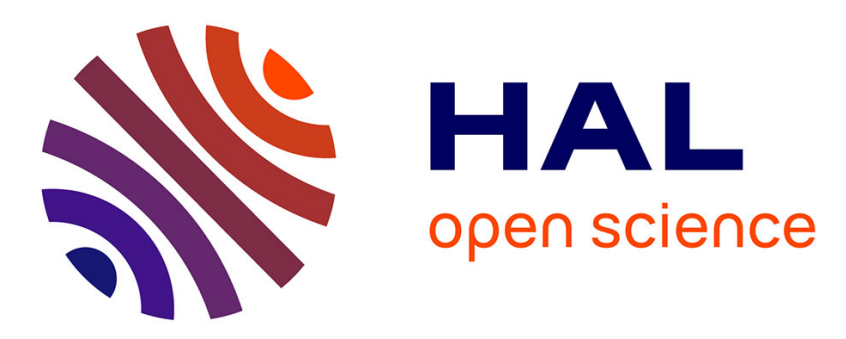

\title{
On the necessity of Nitsche term. Part II: An alternative approach
}

Jean-Paul Boufflet, Marc Dambrine, Gaël Dupire, Pierre Villon

\section{To cite this version:}

Jean-Paul Boufflet, Marc Dambrine, Gaël Dupire, Pierre Villon. On the necessity of Nitsche term. Part II: An alternative approach. Applied Numerical Mathematics, 2012, 5 (62), pp.521-535. 10.1016/j.apnum.2011.12.007 . hal-00731528

\section{HAL Id: hal-00731528 \\ https://hal.science/hal-00731528}

Submitted on 13 Sep 2012

HAL is a multi-disciplinary open access archive for the deposit and dissemination of scientific research documents, whether they are published or not. The documents may come from teaching and research institutions in France or abroad, or from public or private research centers.
L'archive ouverte pluridisciplinaire HAL, est destinée au dépôt et à la diffusion de documents scientifiques de niveau recherche, publiés ou non, émanant des établissements d'enseignement et de recherche français ou étrangers, des laboratoires publics ou privés. 


\title{
On the necessity of Nitsche term. Part II: an alternative approach.
}

\author{
J.P. Boufflet, M. Dambrine†, G. Dupire ${ }^{\ddagger}$ P. Villon ${ }^{\S}$
}

September 2, 2011

\begin{abstract}
The aim of this article is to explore the possibility of using a family of fixed finite elements shape functions that does not match the domain to solve a boundary value problem with Dirichlet boundary condition. The domain is embedded in a bounding box and the finite element approximation is associated to a regular structured mesh of the box. The shape of the domain is independent of the discretization mesh. In these conditions, a meshing tool is never required. This may be especially useful in the case of evolving domains, for examples shape optimization or moving interfaces. Nitsche method has been intensively applied. However, Nitsche is weighted with the mesh size $h$ and therefore is a purely discrete point of view with no interpretation in terms of a continuous variational approach associated with a boundary value problem. In this manuscript, we introduce an alternative to Nitsche method which is associated with a continuous bilinear form. This extension has strong restrictions: it needs more regularity on the data than the usual method. We prove the well-posedness of our formulation and error estimates. We provide numerical comparisons with Nitsche method.
\end{abstract}

\section{Introduction}

The use of non matching regular grids for solving PDE has been explored since a long time. In the case of variational techniques, the main difficulty is to answer at this fundamental question: how to take account of the essential boundary conditions ? One of the first ideas is to use Lagrange multipliers $[3,4]$ or penalty method [5]. This can be coupled with Nitsche method $[18,15]$ to obtain error estimators [22]. This has been successfully applied to domain decomposition [23, 17] and to introduce the fictitious domain method [14]. The drawback of this approach is the ill-conditioning of the optimality system. More recently, combining the X-FEM approximation [24] and the level set technique $[20,21]$ some new approaches have been developed. There are applications in the domain of shape optimization [7], fluid interfaces [8, 9, 10] and stochastic mechanics [19]. What we propose in this article is an alternative way based on a modified bilinear form.

\footnotetext{
*HEUDIASYC, UMR CNRS 6599, Université de Technologie de Compiègne, F-60205 Compiègne, France

${ }^{\dagger}$ LMA, UMR CNRS 5142, Université de Pau et des Pays de l'Adour, F-64013 Pau, France

${ }^{\ddagger}$ LMAC, EA 2222, Université de Technologie de Compiègne, F-60205 Compiègne, France

${ }^{\S}$ ROBERVAL, UMR CNRS 6253, Université de Technologie de Compiègne, F-60205 Compiègne, France
} 
One of the question posed by Nitsche formulation is that it has no sense in a continuous setting: the regularization term depends on the mesh size. In [12], a meshfree method has been introduced that is roughly speaking a unregularized Nitsche method. Let us precise the model problem we will work on. Let $\Omega$ be a domain of $\mathbb{R}^{d}(d \geq 1)$ with a smooth boundary $\partial \Omega$. We consider the following model boundary value problem of Dirichlet type

$$
\left\{\begin{aligned}
-\Delta u & =f \text { in } \Omega \\
u & =g \text { on } \partial \Omega
\end{aligned}\right.
$$

where $f \in \mathrm{L}^{2}(\Omega)$ and $g \in \mathrm{H}^{1 / 2}(\partial \Omega)$. The idea introduced by Dumont and al in [12] and explored here consists in the introduction of the variational problem:

$$
\left\{\begin{array}{l}
\text { Find } v \text { in an appropriate space H such that } \\
B(v, \varphi)=L_{2}(\varphi) \text { for all } \varphi \in \mathrm{H} \text { where } \\
B(v, \varphi)=\int_{\Omega} \nabla u \cdot \nabla \varphi-\int_{\partial \Omega}\left(\varphi \partial_{n} u+u \partial_{n} \varphi\right) \text { and } L_{2}(\varphi)=\int_{\Omega} f \varphi-\int_{\partial \Omega} g \partial_{n} \varphi .
\end{array}\right.
$$

Roughly speaking, this formulation can be seen as a continuous version of a Nitsche method without penalization. Since one should give a sense to the normal derivative, the form $B$ is not defined on $\mathrm{H}^{1}(\Omega)$ but only on $\mathrm{H}_{\Delta}^{1}(\Omega)=\left\{u \in H^{1}(\Omega) / \Delta u \in L^{2}(\Omega)\right\}$. Endowed with the norm $\|\cdot\|_{\mathrm{H}_{\Delta}^{1}(\Omega)}$ defined by

$$
\|u\|_{\mathrm{H}_{\Delta}^{1}(\Omega)}^{2}=\|u\|_{\mathrm{L}^{2}(\Omega)}^{2}+\|\nabla u\|_{\mathrm{L}^{2}(\Omega)}^{2}+\|\Delta u\|_{\mathrm{L}^{2}(\Omega)}^{2},
$$

it is an Hilbert space.

A notable point about $B$ is that this form is not coercive. Elementary computations show that $B(1,1)=0$ and that for any harmonic function $u, B(u, u) \leq 0$. Following Brezzi-BabuskaLadyzhenskaya's theory $([2,3])$, an inf sup argument is used in [12] to prove the well posedness on the continuous level. The former variational problem leads to a discrete method based on a Galerkin approximation built on a grid that does not have to match the domain. A discrete inf sup condition should hold uniformly in order to obtain a robust numerical method, otherwise penalization is mandatory.

In the first part of this work [13], we have discussed this method: convergence is proved in dimension one, the limitations of the method are explored by numerical experiments. In dimension two, these experiments show that the method is unstable on complex geometries and that regularization is then needed. We considered Nitsche method in [13]. Let us briefly recall its basics: the interested reader can find more details in [6]. On the discrete space $V_{h}$, consider the bilinear form $a_{h}$ and the linear form $l_{h}$ defined as

$$
\begin{aligned}
a_{h}^{N}\left(u_{h}, u_{h}\right) & =\int_{\Omega} \nabla u_{h} \cdot \nabla v_{h}-\int_{\partial \Omega}\left(u_{h} \partial_{h} v_{h}+v_{h} \partial_{h} u_{h}\right)+\frac{\beta}{h} \int_{\partial \Omega} u_{h} v_{h}, \\
b_{h}^{N}\left(v_{h}\right) & =\int_{\Omega} f v_{h}-\int_{\partial \Omega} g \partial_{h} v_{h}+\frac{\beta}{h} \int_{\partial \Omega} g v_{h} .
\end{aligned}
$$

The number $\beta$ is a parameter called Nitsche parameter. Nitsche problem can be written as follows:

$$
\left\{\begin{array}{l}
\text { Find } u_{h} \text { in } V_{h} \text { such that } \\
a_{h}^{N}\left(u_{h}, v_{h}\right)=b_{h}^{N}\left(v_{h}\right) \text { for all } v_{h} \in V_{h}
\end{array}\right.
$$


When $\beta$ is large enough, the bilinear form $a_{h}^{N}$ is coercive in the weighted norm $\|\cdot\|_{h}$ defined as

$$
\left\|u_{h}\right\|_{h}^{2}=\left\|\nabla u_{h}\right\|_{\mathrm{L}^{2}(\Omega)}^{2}+\frac{1}{h}\left\|u_{h}\right\|_{\mathrm{L}^{2}(\partial \Omega)}^{2}+h\left\|\nabla u_{h}\right\|_{\mathrm{L}^{2}(\partial \Omega)}^{2} .
$$

In the context of non matching grid, we introduced in [13] a second regularization of geometrical type: a compatibility condition between the geometry and the computational mesh has to be satisfied to obtain good approximation properties. This condition expresses that a uniform lower bound on the size of intersection between the geometries and the regular mesh should hold. Not every domain satisfies this condition. This is nevertheless the case after a small modification of the domain.

In this work, we introduce an alternative way to deal with the essential boundary condition. Our point of view is the following: instead of looking to

$$
u=g \text { on } \partial \Omega,
$$

we consider it after two differentiations

$$
\Delta_{\tau} u=\Delta_{\tau} g \text { on } \partial \Omega
$$

where $\Delta_{\tau}$ is the Laplace-Beltrami operator on the manifold $\partial \Omega$. This is only possible if $g$ is regular enough to give a sense to $\Delta_{\tau} g$. We shall make the assumption that $g \in \mathrm{H}^{1}(\partial \Omega)$ such that $\Delta_{\tau} g$ is defined in $\mathrm{H}^{-1}(\partial \Omega)$.

The manuscript is organized as follows. In Section 2, we first introduce the new formulation based on this idea, then we prove that any solution of this variational problem also solves the original boundary value problem (2.11). In Section 3, we adapt the study of Nitsche method to justify our approach, we obtain existence and uniqueness of the solution to the discretized version of our variational formulation and we obtain error estimates. Finally, in Section 4, we present some numerical experiments to illustrate the proposed method and we provide a numerical comparison of our method with Nitsche method.

\section{A new variational formulation.}

\subsection{Definitions and setting of the problem.}

Let us fix the notations and the variational space. Let $\Omega$ be an open, bounded and smooth subset of $\mathbb{R}^{d}(d \geq 1)$ with a smooth boundary $\partial \Omega$. We first recall some facts of differential calculus on surfaces that will be of great use in the following, the reader can find more details in [16]. The unit outwards normal to $\partial \Omega$ is denoted by $\boldsymbol{n}$. For $u \in \mathcal{C}^{\infty}(\bar{\Omega})$, the tangential gradient of $u$ on $\partial \Omega$ is the function defined as

$$
\nabla_{\tau} u=\nabla u-(\nabla u \cdot \boldsymbol{n}) \boldsymbol{n} .
$$

Then, if $u$ is defined only on $\partial \Omega, \nabla_{\tau} u$ is is defined as $\nabla_{\tau} \tilde{u}$, where $\tilde{u}$ is an arbitrary extension of $u$ since this quantity is independent of the choice of the extension. In particular, the tangential gradient $\nabla_{\tau} u$ of a function $u \in \mathrm{H}^{1}(\partial \Omega)$ belongs to $\mathrm{L}^{2}(\partial \Omega)$. Then, the tangential divergence of a 
vector field $\boldsymbol{v}$ defined on $\partial \Omega$ is the $\mathrm{L}^{2}(\partial \Omega)$-adjoint operator of the tangential gradient. Therefore, it is defined by the relation: for all $u \in \mathcal{C}^{1}(\partial \Omega)$,

$$
\int_{\partial \Omega} \nabla_{\tau} u \cdot \boldsymbol{v}=-\int_{\partial \Omega} u \operatorname{div}_{\tau} \boldsymbol{v}
$$

Finally, the Laplace-Beltrami operator $\Delta_{\tau}$ is defined by $\Delta_{\tau} u=\operatorname{div}_{\tau}\left(\nabla_{\tau} u\right)$ so that we have the following integration by parts formula

$$
\int_{\partial \Omega} \Delta_{\tau} u v=-\int_{\partial \Omega} \nabla_{\tau} u \cdot \nabla_{\tau} v
$$

for a smooth function. Then, this formula extended by density, gives a sense to $\Delta_{\tau} u$ as a element of $\mathrm{H}^{-1}(\partial \Omega)$ for all $u \in \mathrm{H}^{1}(\partial \Omega)$.

A fundamental remark is that no boundary term appears in the former formula (2.10) as long as $\partial \Omega$ is a manifold with no boundary. Formula (2.10) is the keystone of our method. Therefore, the approach we will develop in the following is restricted to the case when the Dirichlet boundary condition is asked on a manifold $\Gamma_{D}$ with no border. This assumption excludes the case of mixed boundary conditions except when the Neumann and Dirichlet conditions are applied on parts $\Gamma_{N}$ and $\Gamma_{D}$ with disjoint closures $\overline{\Gamma_{D}} \cap \overline{\Gamma_{N}}=\emptyset$.

We consider the following model boundary value problem of Dirichlet type

$$
\left\{\begin{aligned}
-\Delta u & =f \text { in } \Omega \\
u & =g \text { on } \partial \Omega,
\end{aligned}\right.
$$

where $f \in \mathrm{L}^{2}(\Omega)$ and $g \in \mathrm{H}^{1}(\partial \Omega)$. We consider the space $\mathrm{H}(\Omega)$ defined by

$$
\mathrm{H}(\Omega)=\left\{u \in \mathrm{H}^{1}(\Omega) \text { such that } \Delta u \in \mathrm{L}^{2}(\Omega) \text { and } u_{\mid \partial \Omega} \in \mathrm{H}^{1}(\partial \Omega)\right\},
$$

where $u_{\mid \partial \Omega}$ denotes the usual trace in $\mathrm{H}^{1 / 2}(\partial \Omega)$ of $u$. Endowed with the norm

$$
\|u\|_{\mathrm{H}(\Omega)}^{2}=\|u\|_{\mathrm{L}^{2}(\Omega)}^{2}+\|\nabla u\|_{\mathrm{L}^{2}(\Omega)}^{2}+\|\Delta u\|_{\mathrm{L}^{2}(\Omega)}^{2}+\|u\|_{\mathrm{L}^{2}(\partial \Omega)}^{2}+\left\|\nabla_{\tau} u\right\|_{\mathrm{L}^{2}(\partial \Omega)}^{2},
$$

the space $H(\Omega)$ is hilbertian with scalar product

$$
\langle u, v\rangle_{\mathrm{H}}=\int_{\Omega}(u v+\nabla u \cdot \nabla v+\Delta u \Delta v)+\int_{\partial \Omega}\left(u v+\nabla_{\tau} u \cdot \nabla_{\tau} v\right) .
$$

\subsection{Derivation of the variational formulation}

Assume that $u$ is the solution of (2.11). Then, multiplying it by a function $\varphi \in \mathrm{H}(\Omega)$ and integrating by parts leads to

$$
\int_{\Omega} \nabla u \cdot \nabla \varphi-\int_{\partial \Omega} \partial_{\boldsymbol{n}} u \varphi=\int_{\Omega} f \varphi .
$$

Now, we use the boundary condition $u=g$ on $\partial \Omega$ to notice that

$$
\int_{\partial \Omega} u \varphi=\int_{\partial \Omega} g \varphi
$$


Then, we use again the boundary condition $u=g$ on $\partial \Omega$ under the form (1.7) to see that

$$
\left\langle\Delta_{\tau} u, \varphi\right\rangle_{\mathrm{H}^{-1}(\partial \Omega) \times \mathrm{H}^{1}(\partial \Omega)}=\left\langle\Delta_{\tau} g, \varphi\right\rangle_{\mathrm{H}^{-1}(\partial \Omega) \times \mathrm{H}^{1}(\partial \Omega)} .
$$

Applying integration by parts formula (2.10), we get

$$
\int_{\partial \Omega} \nabla_{\tau} u \cdot \nabla_{\tau} \varphi=\int_{\partial \Omega} \nabla_{\tau} g \cdot \nabla_{\tau} \varphi
$$

Therefore, combining Equations (2.14)-(2.15) and (2.16), $u$ is a solution to

$$
(\mathcal{P})\left\{\begin{array}{l}
\text { Find } u \text { in } \mathrm{H}(\Omega) \text { such that } A_{\gamma}(u, \varphi)=L_{\gamma}(\varphi) \text { for all } \varphi \in \mathrm{H}(\Omega) \text { where } \\
A_{\gamma}(u, \varphi)=\int_{\Omega} \nabla u \cdot \nabla \varphi-\int_{\partial \Omega}\left(\varphi \partial_{n} u+u \partial_{n} \varphi\right)+\gamma \int_{\partial \Omega} \nabla_{\tau} u \cdot \nabla_{\tau} \varphi \\
L_{\gamma}(\varphi)=\int_{\Omega} f \varphi-\int_{\partial \Omega} g \partial_{n} \varphi+\gamma \int_{\partial \Omega} \nabla_{\tau} g \cdot \nabla_{\tau} \varphi
\end{array}\right.
$$

Here, $\gamma>0$ is a parameter to be chosen later.

Remark 2.1 The quadratic form associated to $A_{\gamma}$ is not coercive: one checks $A_{\gamma}(1,1)=0$ for any value of $\gamma$. Parameter $\gamma$ plays the same role than Nitsche parameter $\beta$ in Nitsche formulation (1.5). Like the weighted tangential mass term

$$
\frac{1}{h} \int_{\partial \Omega} u_{h} v_{h}
$$

in Nitsche method, the tangential rigidity term

$$
\int_{\partial \Omega} \nabla_{\tau} u \cdot \nabla_{\tau} \varphi
$$

is added to compensate, after discretization, the fact that $B$ defined in (1.2) is not coercive in $\mathrm{H}(\Omega)$. Like Nitsche parameter $\beta$, the parameter $\gamma$ is to be chosen large enough to assure some positivity of the bilinear form $A_{\gamma}$ but only once discretized. Like for Nitsche method, the key of the proof that validates this method will be an inverse inequality that provides an upper bound for $\left\|\partial_{\boldsymbol{n}} u_{h}\right\|_{L^{2}(\partial \Omega)}$ in terms of $\left\|\nabla u_{h}\right\|_{\mathrm{L}^{2}(\Omega)}$. We will state it in Lemma 3.2.

\subsection{Equivalence with the boundary value problem.}

Now, we assume that $u \in \mathrm{H}(\Omega)$ solves variational Problem (2.17). We want to characterize $u$ as the solution of a boundary value problem.

Theorem 2.1 If $\gamma>0$, there is equivalence between $u$ solves the variational problem (2.17) and $u$ solves the boundary value problem (2.11). 
Proof: By construction of the variational problem, any solution of boundary value problem (2.11) also solves (2.17). Therefore, it remains to prove that any solution of variational Problem (2.17) also solves boundary value problem (2.11).

We first take any $\varphi \in \mathcal{D}(\Omega)$ as test function in (2.17). Then, by cancellation of $\varphi$ and its derivatives in the neighborhood of $\partial \Omega$, we have

$$
\int_{\Omega} \nabla u \cdot \nabla \varphi=\int_{\Omega} f \varphi
$$

After an integration by parts on $\Omega$, we check that $-\Delta u=f$ in $\Omega$. It remains to check the boundary condition. A reverse integration by parts gives that for any $\varphi \in \mathrm{H}(\Omega)$

$$
\int_{\Omega} \nabla u \cdot \nabla \varphi-\int_{\partial \Omega} \partial_{\boldsymbol{n}} u \varphi=\int_{\Omega} f \varphi
$$

Now, for any $\psi \in \mathcal{C}^{\infty}(\partial \Omega)$, take an extension $\Psi \in \mathrm{H}(\Omega)$ such that $\partial_{n} \Psi=0$ on $\partial \Omega$. Such an extension can by defined by extending $\psi$ as a constant along the orbits of the gradient of the signed distance function to $\partial \Omega$ then multiply it by a cutoff function with respect to the distance to $\partial \Omega$. Then,

$$
A_{\gamma}(u, \Psi)=\int_{\Omega} \nabla u \cdot \nabla \Psi-\int_{\partial \Omega} \psi \partial_{n} u+\gamma \int_{\partial \Omega} \nabla_{\tau} u \cdot \nabla_{\tau} \psi \text { and } L_{\gamma}(\Psi)=\int_{\Omega} f \Psi+\gamma \int_{\partial \Omega} \nabla_{\tau} g \cdot \nabla_{\tau} \psi \text {. }
$$

Therefore, since $\gamma \neq 0$, we have for any $\psi \in \mathcal{C}^{\infty}(\partial \Omega)$

$$
\int_{\partial \Omega} \nabla_{\tau} u \cdot \nabla_{\tau} \psi=\int_{\partial \Omega} \nabla_{\tau} g \cdot \nabla_{\tau} \psi
$$

By integration by parts on $\partial \Omega$, we get by density $-\Delta_{\tau} u=-\Delta_{\tau} g$ in $\mathrm{H}^{-1}(\partial \Omega)$ and that (2.19) also holds for $\psi \in \mathrm{H}^{1}(\partial \Omega)$. Then, $u-g$ is constant on any connected component of $\partial \Omega$ with $\mathcal{H}^{d-1}$ measure defined as $\mathcal{H}^{d-1}(\Gamma)=\int_{\Gamma} 1$.

Fix $\Gamma$ a connected component of $\partial \Omega$. To determine the constant $C_{\Gamma}$ such that $u-g=C_{\Gamma}$ on $\Gamma$, we localize around $\Gamma$ : set $v_{\Gamma}=d_{\Gamma} \chi$ with $d_{\Gamma}$ the signed distance to $\Gamma$ (seen from $\Omega$ ) and $\chi$ a cutoff function with $\chi(x)=1$ in the neighborhood of $\Gamma$ and $\chi(x)=0$ in the neighborhood of the complement of $\Gamma$ in $\partial \Omega$. The function $v_{\Gamma}$ lays in $\mathrm{H}(\Omega)$ and satisfies $v_{\Gamma}=\partial_{\boldsymbol{n}} v_{\Gamma}=0$ on $\partial \Omega \backslash \Gamma$. Then, $A_{\gamma}\left(u, v_{\Gamma}\right)=L_{\gamma}\left(v_{\Gamma}\right)$ reduces by cancellation on the other components of $\partial \Omega$ to

$\int_{\Omega} \nabla u \cdot \nabla v_{\Gamma}-\int_{\Gamma}\left(v_{\Gamma} \partial_{n} u+u \partial_{n} v_{\Gamma}\right)+\gamma \int_{\Gamma} \nabla_{\tau} u \cdot \nabla_{\tau} v_{\Gamma}=\int_{\Omega} f v_{\Gamma}-\int_{\Gamma} g \partial_{n} v_{\Gamma}+\gamma \int_{\Gamma} \nabla_{\tau} g \cdot \nabla_{\tau} v_{\Gamma}$.

Taking into account (2.18) and (2.19), this leads to

$$
0=\int_{\Gamma}(u-g) \partial_{\boldsymbol{n}} v_{\Gamma}=C_{\Gamma} \int_{\Gamma} 1=C_{\Gamma} \mathcal{H}^{d-1}(\Gamma)
$$

Since $\mathcal{H}^{d-1}(\Gamma) \neq 0, C_{\Gamma}=0$ and $u=g$ on $\Gamma$. This can be done connected component by connected component to check $u=g$ on $\partial \Omega$. 


\section{The associated discrete method.}

The computational discretized domain $\Omega_{h}$ is obtained by considering a regular grid (with a square of size $h$ as elementary cell) overlapping $\Omega$ (see Figure 1 ). Then, $\partial \Omega_{h}$ is a polygonal line connecting vertices defined as the intersection points of the boundary $\partial \Omega$ with the grid (see Figure 2).

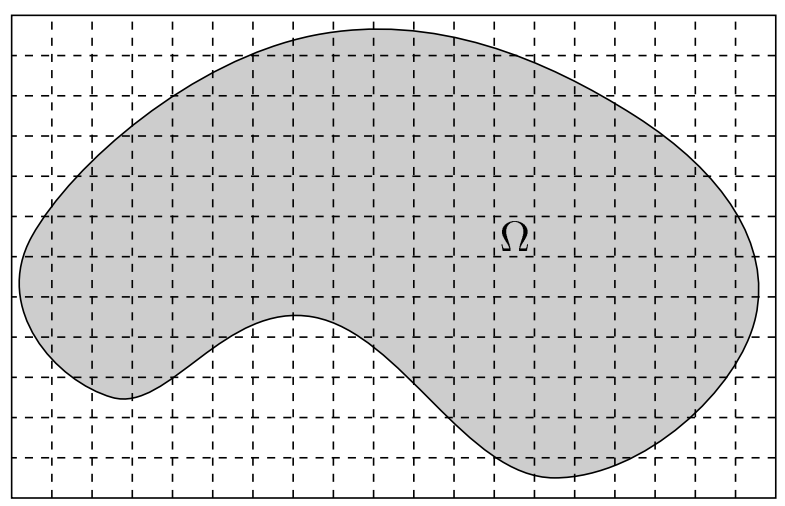

Figure 1: The domain $\Omega$ and the grid.

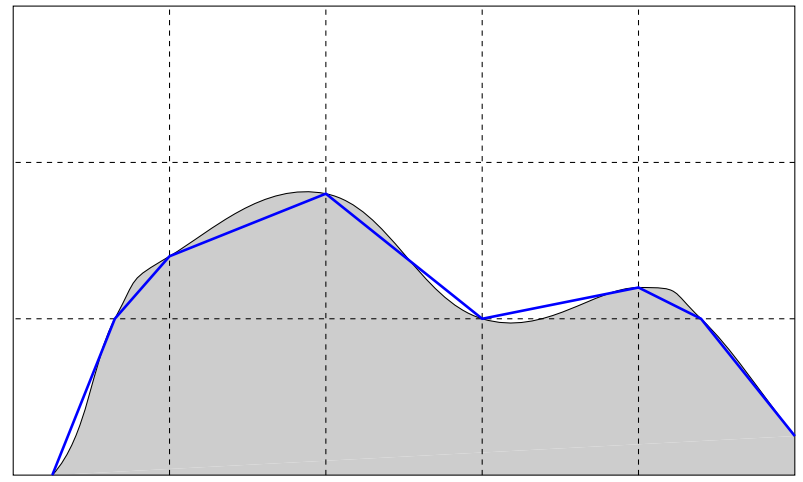

Figure 2: Computational domain $\Omega_{h}$ and original domain $\Omega$.

The regularity properties assumed on $\partial \Omega$ provide upper bounds on the curvature on $\partial \Omega$ (except on possible vertices of $\partial \Omega$ ) so that there exists a constant $C>0$ such that

$$
|\partial \Omega \cap \mathcal{C}| \leq C h,
$$

for each cell $\mathcal{C}$ of size $h$. In the simulations we will present in the sequel, the domain, $\Omega$ is represented as a level line of a function that is discretized on the grid. The vertices of $\partial \Omega_{h}$ are then computed by linear interpolation. As a consequence, $\Omega_{h} \cap \mathcal{C}$ is a polygon as shown on Figure 2 . We choose

$$
V_{h}^{k}=\left\{u \in \mathcal{C}_{0}(\Omega) / u_{\mid K \cap \Omega} \in \mathbb{P}^{k}(K \cap \Omega), \text { for all } K \in \mathcal{T}_{h}\right\},
$$

where $\mathcal{T}_{h}$ denotes the set of the squares of the regular grid and $\mathbb{P}^{k}(K)$ denotes the polynomial's space with degrees less than $k$. To simplify the notations, we will omit the dependency with respect to the degree of approximation $k$ and simply write $V_{h}$ instead of $V_{h}^{k}$. Let us emphasize that $V_{h}$ is not a subset of $\mathrm{H}(\Omega)$ : this comes from the assumption made on the laplacian of function of $\mathrm{H}(\Omega)$. As usually in Nitsche's method, we consider the weighted norm $\|\cdot\|_{h}$ defined on $V_{h}$ as

$$
\left\|u_{h}\right\|_{h}^{2}=\left\|\nabla u_{h}\right\|_{\mathrm{L}^{2}(\Omega)}^{2}+\frac{1}{h}\left\|u_{h}\right\|_{\mathrm{L}^{2}(\partial \Omega)}^{2}+h\left\|\nabla u_{h}\right\|_{\mathrm{L}^{2}(\partial \Omega)}^{2} .
$$

As in [13], we make an additional geometrical assumption in the spirit of the one made for fictitious domain methods or of assumption 2.2 in [6]. In these methods, the size of the surface element should be comparable with the size of the volume element in order to prove convergence. In this work, we make the following geometrical assumption. We consider a cartesian grid of mesh size $h$. 
Definition 3.1 The domain $\Omega$ and the grid satisfy a uniform roundness condition if there exists a constant $\gamma \in(0,1)$ such that, for each cell grid $K$ intersected by the domain $\Omega$,

$$
\rho(K \cap \Omega) \geq \gamma h
$$

where $\rho(E)$ denotes the diameter of the largest disk included in $E$.

As explained in [13], adding this condition provides two keys ingredient: the following interpolation result Proposition 3.1 and the trace lemma 3.1 below quoted from [13].

Proposition 3.1 Let $\Omega$ and a grid satisfying the roundness condition in the sense of Definition 3.1. Let $(K, P, \Sigma)$ be a finite element and let $k, m$ be two integers such that $m<k$. Assume that $\Pi \in \mathcal{L}\left(\mathrm{H}^{k+1}(K), P\right)$ and $P \subset \mathrm{H}^{m}(K \cap \Omega)$. Then, there is a constant $C$ such that

$$
|v-\Pi v|_{m, K \cap \Omega} \leq C h^{k+1-m}|v|_{k+1, \Omega \cap K} .
$$

The proof of Proposition 3.1 is a straightforward adaptation of the usual interpolation result in finite elements theory.

Let us recall the usual Nitsche's formulation: on the discrete space $V_{h}$, consider the bilinear form $a_{h}$ and the linear form $b_{h}$ defined as

$$
\begin{aligned}
a_{h}^{N}\left(u_{h}, u_{h}\right) & =\int_{\Omega} \nabla u_{h} \cdot \nabla v_{h}-\int_{\partial \Omega}\left(u_{h} \partial_{h} v_{h}+v_{h} \partial_{h} u_{h}\right)+\frac{\beta}{h} \int_{\partial \Omega} u_{h} v_{h}, \\
b_{h}^{N}\left(v_{h}\right) & =\int_{\Omega} f v_{h}-\int_{\partial \Omega} g \partial_{n} v_{h}+\frac{\beta}{h} \int_{\partial \Omega} g v_{h} .
\end{aligned}
$$

The number $\beta$ is a parameter called Nitsche parameter. In the approach we follow here, the added surface mass term is changed in a surface rigidity term. On the discrete space $V_{h}$, we consider the bilinear form $a_{h}$ and the linear form $b_{h}$ defined as

$$
\begin{aligned}
a_{h}\left(u_{h}, v_{h}\right) & =\int_{\Omega} \nabla u_{h} \cdot \nabla v_{h}-\int_{\partial \Omega}\left(u_{h} \partial_{h} v_{h}+v_{h} \partial_{h} u_{h}\right)+\frac{\gamma}{h} \int_{\partial \Omega} \nabla_{\tau} u_{h} \cdot \nabla_{\tau} v_{h}, \\
b_{h}\left(v_{h}\right) & =\int_{\Omega} f v_{h}-\int_{\partial \Omega} g \partial_{n} v_{h}+\frac{\gamma}{h} \int_{\partial \Omega} \nabla_{\tau} g \cdot \nabla_{\tau} v_{h} .
\end{aligned}
$$

For reasons that will be explained with Lemma 3.3, we shall work on $V_{h, 0}$, the subspace of $V_{h}$ of the functions with a null mean on the boundary defined as

$$
V_{h, 0}=\left\{u \in \mathcal{C}_{0}(\Omega) / u_{\mid K \cap \Omega} \in \mathbb{P}^{k}(K \cap \Omega), \text { for all } K \in \mathcal{T}_{h} \text { such that } \int_{\partial \Omega} u_{h}=0\right\} .
$$

Therefore, we restrict ourselves to right hand side $g$ of zero mean value. Let us emphasize that this is not a restriction thanks to the linearity of the Laplace operator. Let $g$ be any function of $\mathrm{H}^{1}(\partial \Omega)$, define its mean value

$$
[g]=\frac{1}{|\partial \Omega|} \int_{\partial \Omega} g
$$


and make the change of unknown $w=u-[g]$. Then, $w$ is the unique solution of the boundary value problem

$$
\left\{\begin{aligned}
-\Delta w & =f \text { in } \Omega, \\
w & =g-[g] \text { on } \partial \Omega,
\end{aligned}\right.
$$

with a right hand side $g-[g]$ of zero mean value. Hence, to solve the original boundary value problem (1.1), it suffices to compute the mean value $[g]$ of $g$ then to solve the modified boundary value problem (3.29).

The discrete variational problem on $V_{h, 0}$ can be written as follows :

$$
\left(\mathcal{P}_{h, 0}\right) \text { Find } u_{h} \text { in } V_{h, 0} \text { such that } a_{h}\left(u_{h}, v_{h}\right)=b_{h}\left(v_{h}\right) \text { for all } v_{h} \in V_{h, 0}
$$

To study this problem, we follow the usual steps in the proof of convergence for Nitsche method: we first prove trace and inverse inequalities, then we show that $a_{h}$ is coercive in the norm $\|\cdot\|_{h}$ and finally we conclude by the error estimate.

Lemma 3.1 There is a non negative constant $C_{1}$ such that, for any function $u_{h}$ in $V_{h}$,

$$
\left\|u_{h}\right\|_{\mathrm{L}^{2}(\partial \Omega)}^{2} \leq \frac{C_{1}}{h}\left\|u_{h}\right\|_{\mathrm{L}^{2}(\Omega)}^{2},
$$

We refer to [13] for the proof: the geometrical condition is needed to justify that $C_{1}$ is not domain and mesh size dependent. Since $\partial_{n} u_{h}=\nabla u_{h} \cdot \boldsymbol{n}$, Pythagore's equality provides the following inverse inequality result as a direct consequence of (3.31) applied to $\nabla u$.

Lemma 3.2 There is a non negative constant $C$ such that, for any function $u_{h} \in V_{h}$,

$$
\left\|\partial_{n} u_{h}\right\|_{\mathrm{L}^{2}(\partial \Omega)}^{2} \leq \frac{C}{h}\left\|\nabla u_{h}\right\|_{\mathrm{L}^{2}(\Omega)}^{2} \text { and }\left\|\nabla_{\tau} u_{h}\right\|_{\mathrm{L}^{2}(\partial \Omega)}^{2} \leq \frac{C}{h}\left\|\nabla u_{h}\right\|_{\mathrm{L}^{2}(\Omega)}^{2} .
$$

Finally, to deal with the last term of $a_{h}$, we need a control of the $\mathrm{L}^{2}(\partial \Omega)$ norm of $u$ by the $\mathrm{L}^{2}(\partial \Omega)$ norm of its tangential gradient $\nabla_{\tau} u$. Obviously, this is not possible for every function as shown by the example of the constant function. This is why we imposed in this first step the restriction

$$
\int_{\partial \Omega} u_{h}=0
$$

and work only on $V_{h, 0}$. The control of $\left\|u_{h}\right\|_{\mathrm{L}^{2}(\partial \Omega)}^{2}$ is classically obtained by Wirtinger inequality (in dimension two, the boundary is a curve).

Lemma 3.3 There is a non negative constant $C_{W}$ that depends on $\Omega$ such that, for any function $u_{h} \in V_{h, 0}$,

$$
\left\|u_{h}\right\|_{\mathrm{L}^{2}(\partial \Omega)} \leq C_{W}\left\|\nabla_{\tau} u_{h}\right\|_{\mathrm{L}^{2}(\partial \Omega)} .
$$


Let us sketch the proof of this lemma. In dimension two, the boundary is a curve, $u_{h}$ is a periodic function and the proof is a direct consequence of Parseval formula for Fourier series. In higher dimension, things are not elementary. Following [25], the Laplace-Beltrami operator $-\Delta_{\tau}$ on $\partial \Omega$ is a positive, formally self-adjoint, elliptic pseudo-differential operator of real symbol of order two for which the spectrum (as an unbounded operator on $\mathrm{H}^{s}(\partial \Omega)$ ) is independent of $\mathrm{s}$ and is made of a series of real eigenvalues growing to infinity. Its kernel is the space of constant functions to which $V_{h, 0}$ is $\mathrm{L}^{2}(\partial \Omega)$ orthogonal, hence the Courant Fischer formulae for the Rayleigh quotient of the Laplace Beltrami operator provides the result.

Let emphasize that this control is not an inverse inequality: it is based on global arguments. One could ask if a better estimate with a gain in $h$ holds. It is an open question: it is true on each element but the global property of null mean value plays a crucial role and cannot be handled easily at the element level. This fact is the main obstacle to a unweighted discretization of the variational formulation studied in Section 2.

We now prove that the quadratic form $a_{h}$ defined in (3.26) is coercive in the weighted norm $\|\cdot\|_{h}$ for $\gamma$ large enough. Set $\gamma_{0}=C C_{W}$ where $C$ is the constant given by the inverse inequality, Lemma 3.2 and $C_{W}$ the constant in Wirtinger's lemma.

Proposition 3.2 If $\gamma>\gamma_{0}$, there is a non negative constant $\alpha$ independent of $h$ but not of $\gamma$ such that for all $u_{h} \in V_{h, 0}$

$$
a_{h}\left(u_{h}, u_{h}\right) \geq \alpha\left\|u_{h}\right\|_{h}^{2} .
$$

Then, the discrete problem $\left(\mathcal{P}_{h, 0}\right)$ defined in (3.30) has a unique solution.

Proof: By Cauchy-Schwarz inequality, one has

$$
\begin{aligned}
a_{h}\left(u_{h}, u_{h}\right) & =\int_{\Omega}\left|\nabla u_{h}\right|^{2}-2 \int_{\partial \Omega} u_{h} \partial_{n} u_{h}+\frac{\gamma}{h} \int_{\partial \Omega}\left|\nabla_{\tau} u_{h}\right|^{2} \\
& \geq\left\|\nabla u_{h}\right\|_{L^{2}(\Omega)}^{2}-2\left\|h^{-1 / 2} u_{h}\right\|_{L^{2}(\partial \Omega)}\left\|h^{1 / 2} \partial_{n} u_{h}\right\|_{L^{2}(\partial \Omega)}+\frac{\gamma}{h}\left\|\nabla_{\tau} u_{h}\right\|_{L^{2}(\partial \Omega)}^{2}
\end{aligned}
$$

We introduce a parameter $\varepsilon$ to be fixed later. By Cauchy inequality, we have

$$
a_{h}\left(u_{h}, u_{h}\right) \geq\left\|\nabla u_{h}\right\|_{L^{2}(\Omega)}^{2}-\varepsilon\left\|h^{-1 / 2} u_{h}\right\|_{L^{2}(\partial \Omega)}^{2}-\frac{1}{\varepsilon}\left\|h^{1 / 2} \partial_{h} u_{h}\right\|_{L^{2}(\partial \Omega)}^{2}+\frac{\gamma}{h}\left\|\nabla_{\tau} u_{h}\right\|_{L^{2}(\partial \Omega)}^{2} .
$$

Then, by the inverse inequality (3.32) and Wirtinger inequality (3.33), we get

$$
\begin{aligned}
a_{h}\left(u_{h}, u_{h}\right) & \geq \frac{\varepsilon-C}{\varepsilon}\left\|\nabla u_{h}\right\|_{\mathrm{L}^{2}(\Omega)}^{2}+\frac{\gamma}{h}\left\|\nabla_{\tau} u_{h}\right\|_{\mathrm{L}^{2}(\partial \Omega)}^{2}-\frac{\varepsilon}{h}\left\|u_{h}\right\|_{\mathrm{L}^{2}(\partial \Omega)}^{2}, \\
& \geq \frac{\varepsilon-C}{\varepsilon}\left\|\nabla u_{h}\right\|_{\mathrm{L}^{2}(\Omega)}^{2}+\frac{\gamma-C_{W} \varepsilon}{h}\left\|\nabla_{\tau} u_{h}\right\|_{\mathrm{L}^{2}(\partial \Omega)}^{2}, \\
& \geq \frac{\varepsilon-C}{\varepsilon}\left\|\nabla u_{h}\right\|_{\mathrm{L}^{2}(\Omega)}^{2}+\frac{\gamma-C_{W} \varepsilon}{C_{W} h}\left\|u_{h}\right\|_{\mathrm{L}^{2}(\partial \Omega)}^{2} .
\end{aligned}
$$

Now, we first take $\varepsilon>C$ and then $\gamma>C_{W} \varepsilon$, hence $\gamma_{0}$ can be chosen as $C C_{W}$. We have proven the coercivity result (3.34) for $\left.\alpha=\min \left((\varepsilon-C) / \varepsilon,\left(\gamma-C_{W} \varepsilon\right) / C_{W}\right)\right)>0$. 
The continuity of the forms is automatic in the finite dimensional context: nevertheless, the constants of continuity depend on $h$ and of $\gamma$. Here, using Lemma 3.2 to dominate the surface rigidity term or directly the definition of Nitsche's norm (3.21), we have only the poor

$$
\left|a_{h}\left(u_{h}, v_{h}\right)\right| \leq C_{1} h^{-2}\left\|u_{h}\right\|_{h}\left\|v_{h}\right\|_{h} .
$$

Next lemma, stating Galerkin orthogonality, will be used to prove the convergence.

Lemma 3.4 Consider (2.11) with the condition $[g]=0$. Let $u$ be its solution and let $u_{h}$ be the solution of the discrete problem (3.30) then $a_{h}\left(\left(u_{h}-u\right), \varphi_{h}\right)=0$ for all $\varphi_{h} \in V_{h}$.

Proof: $\quad$ Fix $\varphi_{h} \in V_{h, 0}$ and compute:

$$
\begin{aligned}
a_{h}\left(u, \varphi_{h}\right) & =\int_{\Omega} \nabla u \cdot \nabla \varphi_{h}-\int_{\partial \Omega}\left(\varphi_{h} \partial_{n} u+u \partial_{n} \varphi_{h}\right)+\frac{\gamma}{h} \int_{\partial \Omega} \nabla_{\tau} u \cdot \nabla_{\tau} \varphi_{h} \\
& =\int_{\Omega} \nabla u \cdot \nabla \varphi_{h}-\int_{\partial \Omega} \varphi_{h} \partial_{n} u-\int_{\partial \Omega} g \partial_{n} \varphi_{h}+\frac{\gamma}{h} \int_{\partial \Omega} \nabla_{\tau} g \cdot \nabla_{\tau} \varphi_{h} .
\end{aligned}
$$

Since $V_{h} \subset \mathrm{H}^{1}(\Omega)$, we can integrate by parts and we find:

$$
\begin{aligned}
a_{h}\left(u, \varphi_{h}\right) & =-\int_{\Omega} \varphi_{h} \Delta u-\int_{\partial \Omega} g \partial_{n} \varphi_{h}+\frac{\gamma}{h} \int_{\partial \Omega} \nabla_{\tau} g \cdot \nabla_{\tau} \varphi_{h} \\
& =\int_{\Omega} f \varphi_{h}-\int_{\partial \Omega} g \partial_{h} \varphi_{h}+\frac{\gamma}{h} \int_{\partial \Omega} \nabla_{\tau} g \cdot \nabla_{\tau} \varphi_{h} .
\end{aligned}
$$

The conclusion follows by considering $u_{h} \in V_{h}$, the solution of the discrete problem (3.30) which satisfies:

$$
a_{h}\left(u_{h}, \varphi_{h}\right)=\int_{\Omega} f \varphi_{h}-\int_{\partial \Omega} g \partial_{n} \varphi_{h}+\frac{\gamma}{h} \int_{\partial \Omega} \nabla_{\tau} g \cdot \nabla_{\tau} \varphi_{h} .
$$

We now assume that the uniform roundness compatibility condition is satisfied in order to have Proposition 3.1 at our disposal. We can state the error estimate.

Theorem 3.1 Consider (2.11) with the condition $[g]=0$. Assume $\gamma>\gamma_{0}$. Let $u$ be its solution and let $u_{h}$ be the solution of problem (3.30). If $u \in \mathrm{H}^{s}(\Omega)$ with $2 \leq s \leq k$, and assume that the uniform roundness condition (3.22) is satisfied. Then, there is $C>0$ such that:

$$
\left\|u-u_{h}\right\|_{h} \leq C h^{s-3}\|u\|_{\mathrm{H}^{s}(\Omega)} .
$$


Proof: We proceed in the usual way. For any function $\varphi_{h} \in V_{h}$, we get by triangular inequality

$$
\left\|u-u_{h}\right\|_{h} \leq\left\|u-\varphi_{h}\right\|_{h}+\left\|u_{h}-\varphi_{h}\right\|_{h} .
$$

Using the continuity and coercivity of $a_{h}(3.34)$ and (3.35), and also Lemma 3.4 , there is $C_{1}>0$ such that:

$$
\left\|u_{h}-\varphi_{h}\right\|_{h}^{2} \leq \frac{1}{\alpha} a_{h}\left(u_{h}-\varphi_{h}, u_{h}-\varphi_{h}\right) \leq \frac{1}{\alpha} a_{h}\left(u_{h}-\varphi_{h}, u-\varphi_{h}\right) \leq \frac{C_{1}}{\alpha} h^{-2}\left\|u_{h}-\varphi_{h}\right\|_{h}\left\|u-\varphi_{h}\right\|_{h} .
$$

Gathering the two inequalities, we get

$$
\left\|u_{h}-\varphi_{h}\right\|_{h} \leq\left(1+\frac{C_{1}}{\alpha} h^{-2}\right) \inf _{\varphi_{h} \in V_{h}}\left\|u-\varphi_{h}\right\|_{h} .
$$

We introduce the trianglewise defined norm :

$$
\|\varphi\|_{h, \mathrm{H}^{2}(\Omega)}^{2}=\sum_{K \in \mathcal{T}_{h}}\|\varphi\|_{\mathrm{H}^{2}(\Omega \cap K)}^{2} .
$$

to recall the following upper bound of the weighted norm proven in [26] for example: there is $C_{2}>0$ such that, for any function $\varphi \in \mathrm{H}^{2}(\Omega)$ :

$$
\|\varphi\|_{h} \leq C_{2} h^{-1}\left(\|\varphi\|_{\mathrm{L}^{2}(\Omega)}+h\|\varphi\|_{\mathrm{H}^{1}(\Omega)}+h^{2}\|\varphi\|_{h, \mathrm{H}^{2}(\Omega)}\right) .
$$

Thanks to Proposition 3.1 on the local approximation, there is a constant $C_{3}>0$ such that :

$$
\|u-\Pi(u)\|_{\mathrm{L}^{2}(\Omega)}+h\|u-\Pi(u)\|_{\mathrm{H}^{1}(\Omega)}+h^{2}\|u-\Pi(u)\|_{h, \mathrm{H}^{2}(\Omega)} \leq C_{3} h^{s}\|u\|_{\mathrm{H}^{s}(\Omega)} .
$$

Combining (3.38) with (3.39), we obtain

$$
\inf _{\varphi_{h} \in V_{h}}\left\|u-\varphi_{h}\right\|_{h} \leq C_{2} C_{3} h^{s-1}\|u\|_{\mathrm{H}^{s}(\Omega)} .
$$

We deduce the convergence estimate (3.36) from the upper bounds (3.37) and (3.40).

The poor scaling of the surface diffusion and the choice of keeping the weighted norm of Nitsche's method explain why we have the poor convergence result: convergence is proved only if $s>3$ with also $k \geq 4$ at least. However, this unsatisfactory theoretical result is not observed in numerical tests as presented in next section.

\section{Numerical validation and comparison with Nitsche method.}

\subsection{Numerical implementation.}

Not every domain satisfies the compatibility condition of Definition (3.1), for example this is the case of a starfish like domain as illustrated in Figure 6. In order to force the compatibility condition, we have to modify the domain $\Omega$ by moving its boundary points that are too close from grid vertices. 
Since the roundness condition (3.1) is not easily checked, we have introduced in [13] a stronger compatibility condition that would be easy to check in implementation. We consider a regular grid $G_{h}$ of size $h$ covering the domain $\Omega$. Each cell $K$ of the grid is then a cube of side $h$, its border $\partial K$ has vertices $S$ that are the nodes of the grid. We set $\partial K^{*}$ the border $\partial K$ from which we removed the vertices. The union of the $\partial K^{*}$ is denoted by $G^{*}$.

Definition 4.1 The domain $\Omega$ and the grid are said to be compatible if there exists a constant $P_{c} \in(0,1 / 2)$ such that if $S \notin \partial \Omega$ is a node of the grid, then

$$
\|S-Y\| \geq P_{c} h \text { for all } Y \in \partial \Omega \cap G^{*} .
$$

The key point is (4.42) that plays the same role than the roundness of the element in the usual result. The choice of the stronger condition (4.41) in Definition 4.1 is motivated by practical consideration: it provides an easily implemented way to modify the domain in order to check the needed (4.42). Let us explain how to perform that modification.

Figure 3 illustrates the forbidden places for boundary points of $\partial \Omega$. Once this property is satisfied, then the diameter $\rho(K \cap \Omega)$ of the largest disk included in $\Omega \cap K$ is uniformly in $h$ controlled from below. In dimension two, the set $K \cap \Omega$ contains a least an isosceles triangle of size length $P_{c} h$, $P_{c} h$ and $\sqrt{2} P_{c} h$ and therefore:

$$
\rho(K \cap \Omega) \geq \frac{1}{2} P_{c} h .
$$

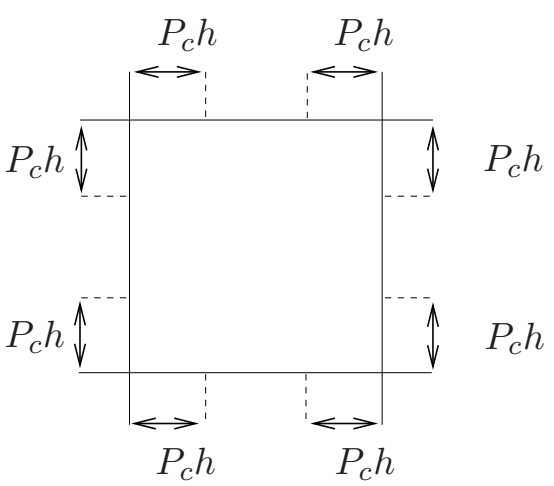

Figure 3: illustration of Definition 4.1 on a cell $K$ of size $h$ of the grid.

This is performed as illustrated in Figures 4 and 5. The domain $\Omega$ is hatched. For computations, it is modified: the black area is either removed from $\Omega$, or added to $\Omega$ and a computational domain $\tilde{\Omega}_{h}$ is obtained. By an argument of continuity and derivability with respect to the domain of the solution in $\mathrm{H}^{1}$ of $(2.11)$, the error on the solution $\tilde{u}_{h}$ committed by this geometrical modification is small (of order $P_{c} h^{2}$ ). We refer to the monographs $[1,16]$ on the subject.

According to the numerical study for the choice of this parameter performed in [13], we have fixed the value of parameter $P_{c}=1 / 10$.

Finally, the tangential gradients are computed thanks to (2.8): on each cell $K$, the boundary $\partial \Omega \cap K$ is a straight line and the tangential gradient is nothing but the component of the gradient in the 


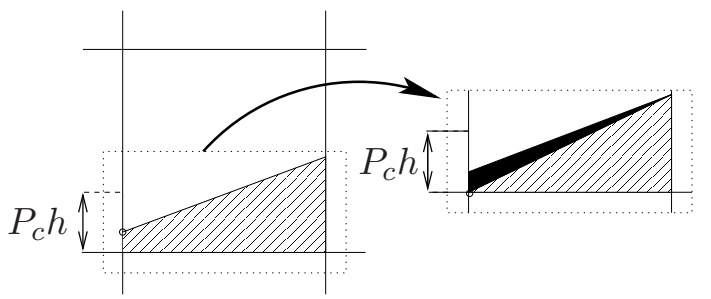

Figure 4: case where $\|S-Y\|<P_{c} h / 2$. The dark area is removed.

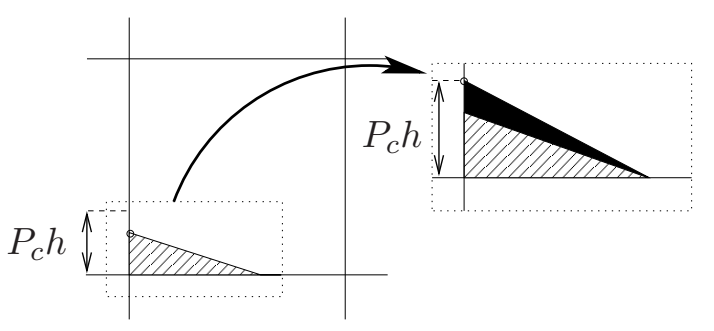

Figure 5: case where $\|S-Y\| \geq P_{c} h / 2$. The dark area is added.

direction of this line. One has to be careful with the orientation. The surface rigidity term is then assembled in a loop over all cells intersected by $\partial \Omega$.

\subsection{Numerical results and comparison with Nitsche method}

Our theoretical results are valid only for boundary values with zero mean value, so we solve (2.11). The presented computations have been performed on two domains: a matching square $[-0.4,0.4] \times$ $[-0.4,0.4]$, and on a more complex domain: the interior of the curve given in polar coordinates by $\rho(\theta)=0.385+0.09 \cdot \cos (7 \cdot \theta+(7 / \pi))$. This domain looks like a starfish as it is shown in Figure 6. Software was developed using MATLAB (The MathWorks). Let us emphasize that the square, with just a Lipschitz boundary, does not fit the assumptions of this work.

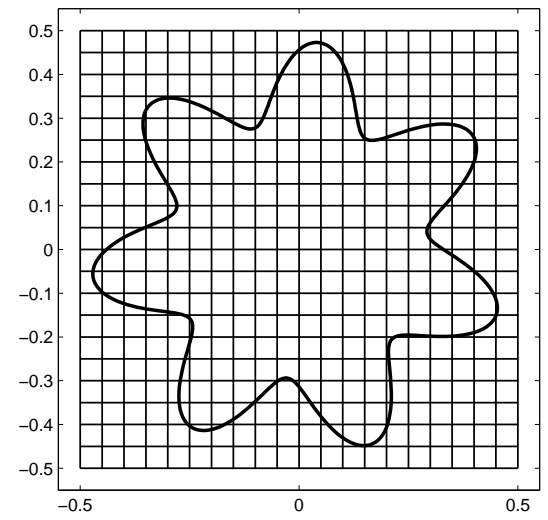

Figure 6: A domain as a starfish plotted on a grid $(h=1 / 20)$.

The sample steps $h=1 / 400,1 / 220,1 / 120,1 / 70,1 / 40,1 / 20,1 / 10$ have been chosen so that the square exactly matches the regular grid composed of $Q 8$-finite elements. The order two is not justified by Theorem 3.1 that suggests to use higher order elements. Nevertheless, we will see that convergence will be observed with an order much better than expected. This suggests that our theoretical analysis can be improved. 
The exact solution we seek to recover is $u(x, y)=e^{y} \sin x$. Intensive tests have been performed ranging $\gamma$ from small values (i.e. $\gamma \in\{1,2,3,4,5\}$ ) to very large value (up to 200). Experiments for $\gamma=5$ to 200 have been conduced using a step of 5 . The geometrical parameter $P_{c}$ has been set to $10 \%$ as it seems to be a good choice referring to the previous experiments presented in [13]. From this field of investigation, we comment on the observed results.

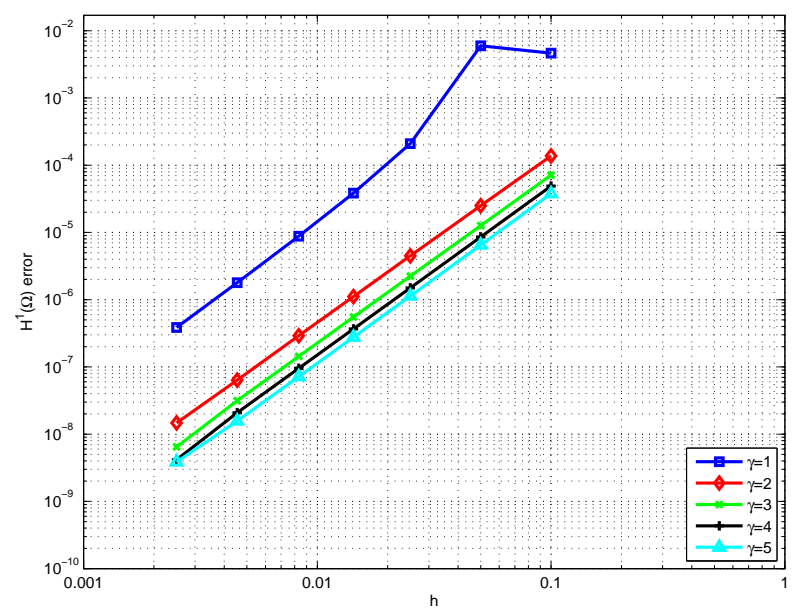

Figure 7: $\mathrm{H}^{1}$ error for the matching square: $\gamma \in\{1,2,3,4,5\}$

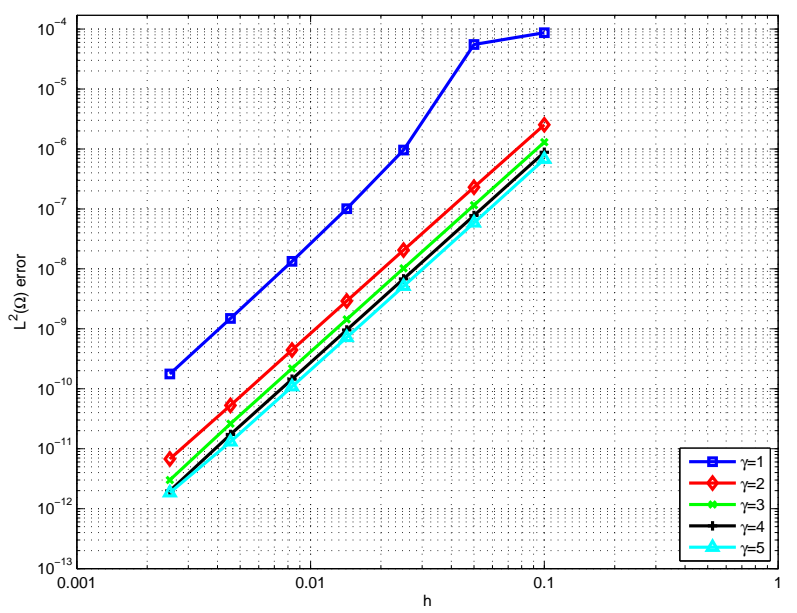

Figure 8: $\mathrm{L}^{2}$ error for the matching square: $\gamma \in\{1,2,3,4,5\}$

Figures 7 to 10 show $\mathrm{H}^{1}$ and $\mathrm{L}^{2}$ errors as $h$ for the matching square for small values of parameter $(\gamma \in\{1,2,3,4,5\})$, and for larger values $(\gamma \in[5,25]$ stepped by 5$)$. Figures 11 to 14 show $\mathrm{H}^{1}$ and $\mathrm{L}^{2}$ errors as $h$ for the starfish for small values of parameter $(\gamma \in\{1,2,3,4,5\})$, and for larger values $(\gamma \in[5,25]$ stepped by 5$)$. For each domain we present the convergence graphs for $\mathrm{H}^{1}$ and $\mathrm{L}^{2}$ errors as $h$ plotted using loglog scales.

It can be observed that, for the same value of $\gamma$, convergence graphs for $\mathrm{L}^{2}$ errors have the same behavior as the convergence graphs for $\mathrm{H}^{1}$ errors. This has been noted on all the experiments.

Figures 15 to 16 depict convergence graphs for $\mathrm{H}^{1}$ error only using $\gamma \in[30,50]$ stepped by 5 for the matching square and for the starfish. As it can be observed, the curves are more smoothed as larger values for $\gamma$ are used.

As it can be observed in Figures 7-10 and 15 a quasi straight line for convergence graphs is obtained when $\gamma$ exceeds 2 for the matching square. If now consider Figures 11-14 and 16, we observe that a good behavior is obtained when $\gamma$ exceeds 5 for the starfish.

As it can be shown on all Figures, small values of $\gamma$ suffice to ensure a good enough convergence. So, we do not present the results obtained for large values of $\gamma$ even though experiments have been conduced up to 200. At this stage, we are not able to provide a safe recipe for choosing $\gamma$ since only two domains have been used for our experiments.

From the graphs presented in Figures 7-16, we see that the value of parameter $\gamma$ has to be chosen 


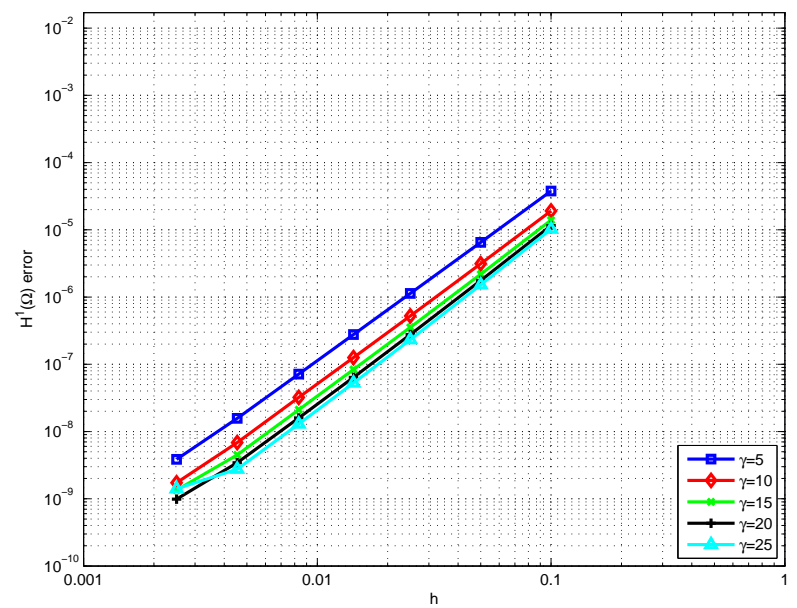

Figure 9: $\mathrm{H}^{1}$ error for the matching square: $\gamma \in[5,25]$

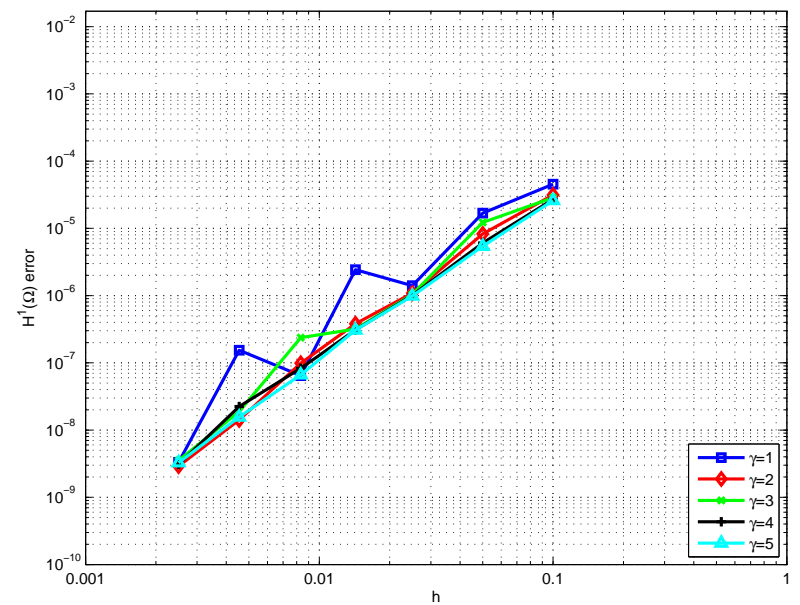

Figure 11: $\mathrm{H}^{1}$ error for the starfish: $\gamma \in[1,5]$

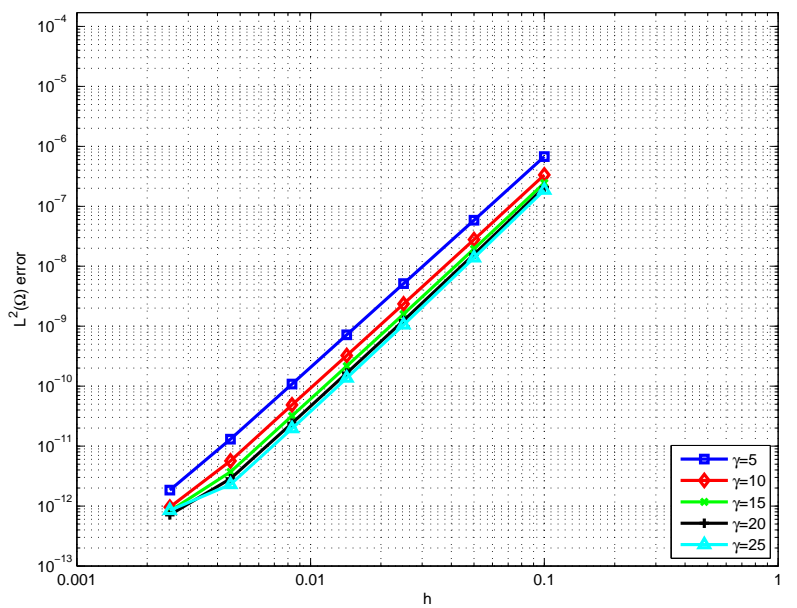

Figure 10: $\mathrm{L}^{2}$ error for the matching square: $\gamma \in[5,25]$

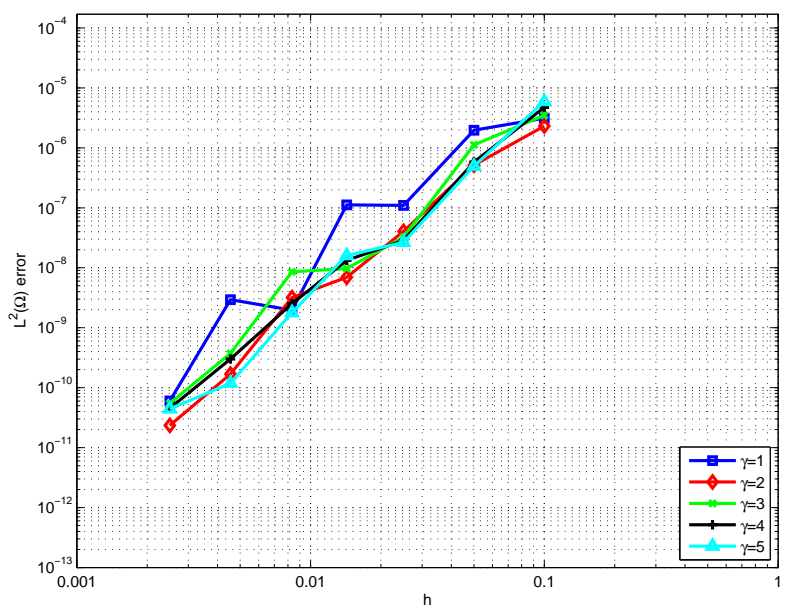

Figure 12: $\mathrm{L}^{2}$ error for the starfish: $\gamma \in[1,5]$

relatively small. This is remarkable in comparison with the usual Nitsche method we used in [13] where the value of the parameter $\beta$ is usually larger. Let us exhibits this observation on the starfish domain. Figure 17 shows $\mathrm{H}^{1}$ error for the same value of the parameter $\gamma=\beta=10$ while Figure 18 depicts results for $\gamma=10$ and $\beta=100$.

At this stage, we have not an explanation about the fact that $\gamma$ can be affected a smaller value than the Nitsche's parameter $\beta$. We have just observed this fact on the performed experiments.

We are now giving some details about the practical implementation. We use MATLAB and standard double-precision having a floating-point relative accuracy of eps $=2.2204 e-16$ around 1.0. We 


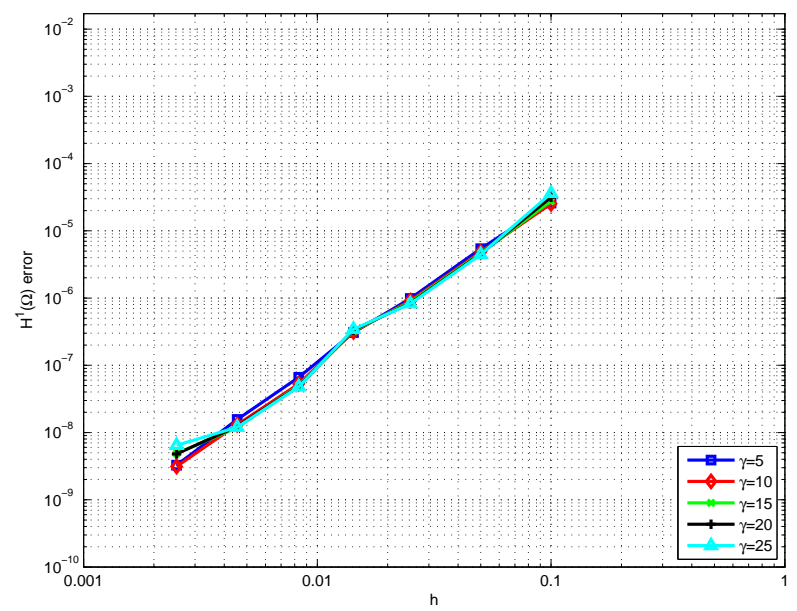

Figure 13: $\mathrm{H}^{1}$ error for the starfish: $\gamma \in[5,25]$

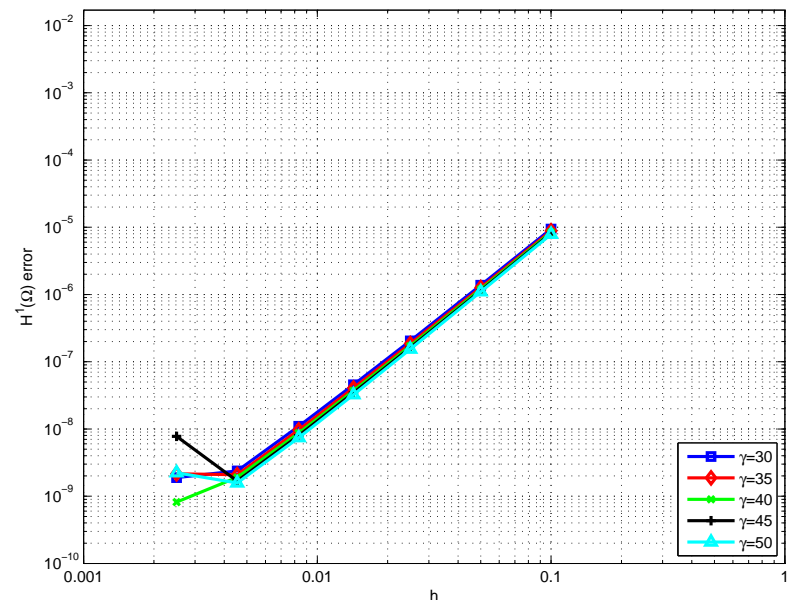

Figure 15: $\mathrm{H}^{1}$ error for the matching square: $\gamma \in[30,50]$

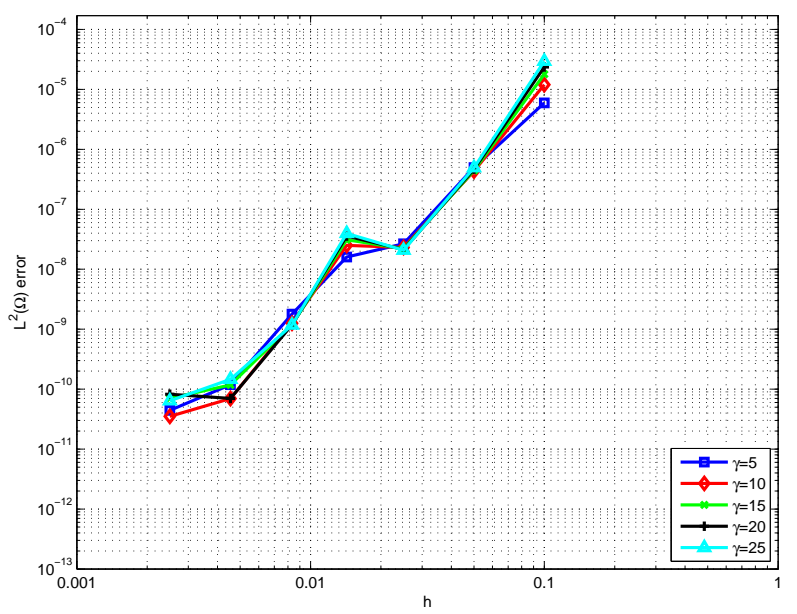

Figure 14: $\mathrm{L}^{2}$ error for the starfish: $\gamma \in[5,25]$

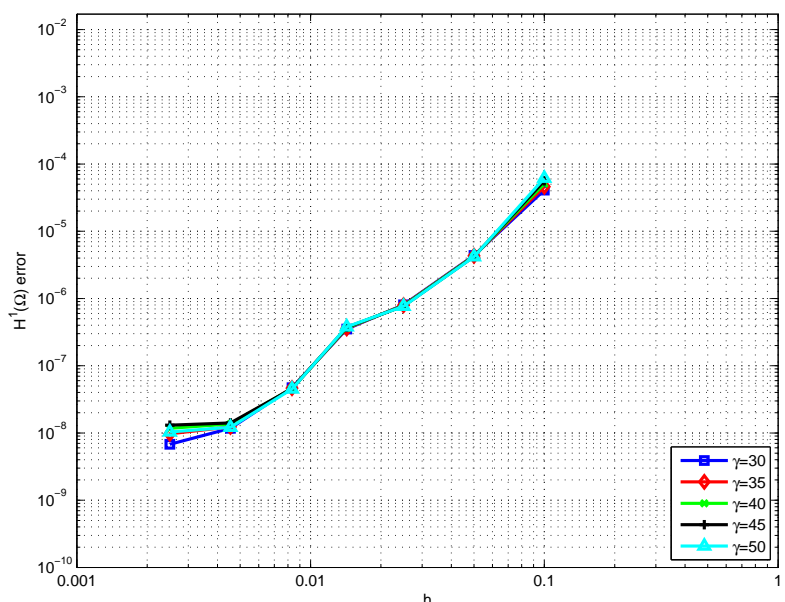

Figure 16: $\mathrm{H}^{1}$ error for the starfish like domain: $\gamma \in[30,50]$

use the UMFPACK direct method [11] of MATLAB and the embedded default ordering.

Tables 1 and 2 show the characteristics of the matrices associated with each problem to be solved. As expected, these matrices become as large as $h$ decreases.

Figures 19 and 20 depict the condition number estimate for matrices for the matching square while Figures 21 and 22 show the condition number estimate for the starfish. For Figures 19 and 21 we have $\gamma \in\{1,2,3,4,5\}$. The two other Figures are plotted using $\gamma \in[5,25]$ stepped by 5 . The condest function of MATLAB has been used to obtain the values. These Figures clearly show that matrices are as much as ill-conditioned as $h$ decreases since the size of the linear system becomes 


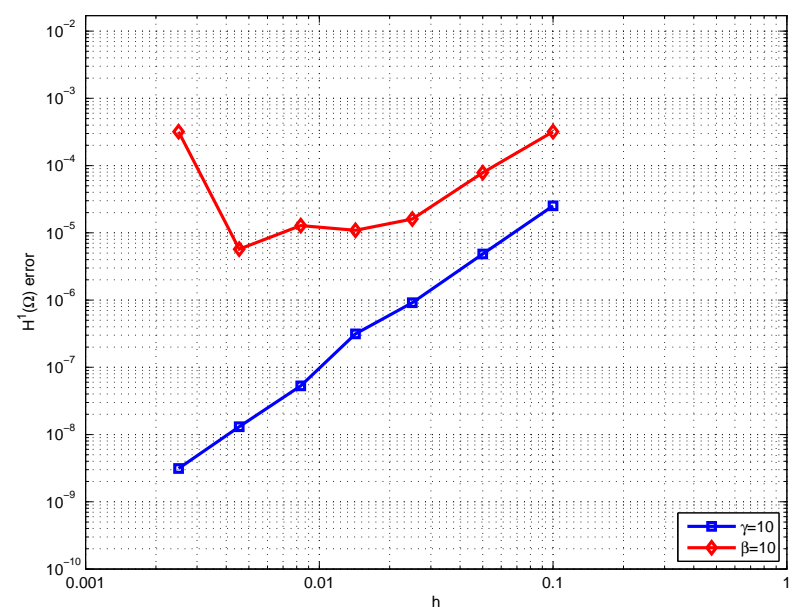

Figure 17: $\mathrm{H}^{1}$ error for the starfish like domain: $\gamma=\beta=10$

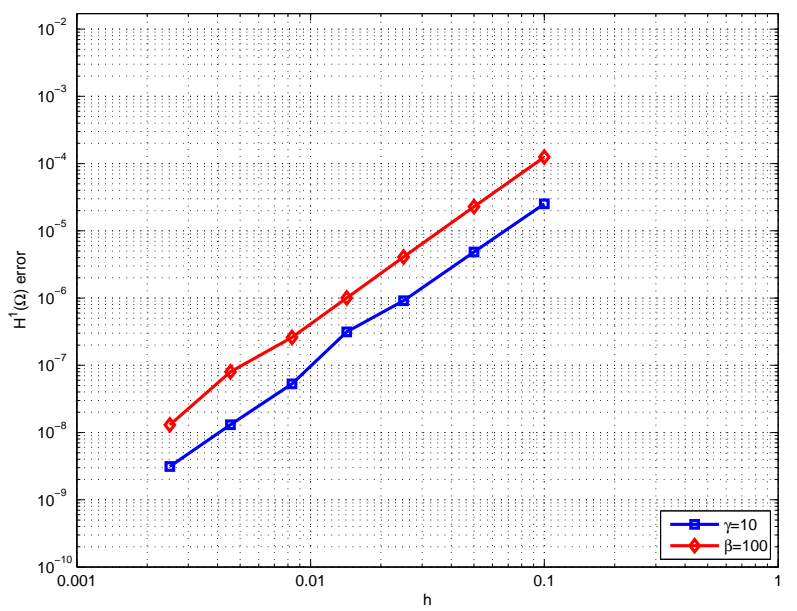

Figure 18: $\mathrm{H}^{1}$ error for the starfish like domain: $\gamma=10$ and $\beta=100$

\begin{tabular}{|l|r|r|r|r|r|r|r|}
\hline$h$ & $1 / 10$ & $1 / 20$ & $1 / 40$ & $1 / 70$ & $1 / 120$ & $1 / 220$ & $1 / 400$ \\
\hline \hline size & 225 & 833 & 3201 & 9633 & 28033 & 93633 & 308481 \\
\hline nnz & 3133 & 12285 & 48633 & 148289 & 434689 & 1458689 & 4817919 \\
\hline sparsity & $6.1 \%$ & $1.7 \%$ & $0.47 \%$ & $0.15 \%$ & $0.055 \%$ & $0.016 \%$ & $0.0050 \%$ \\
\hline
\end{tabular}

Table 1: Size, number of non zero entries, and sparsity rate as $h$ for the matching square domain

larger and larger. Moreover, one can observe that the condition number increases as the parameter $\gamma$ increases. So, pro and cons have to be weighted, oscillations of the solutions are smoothed adding the stabilization term, but as a counterpart the condition number increases.

One can also observe on Figure 15 that curves diverge when $h$ is below 0.01 . This phenomenon has been seen over the other experiments. At this stage, we have not a fully satisfactory explanation. The MATLAB relative floating-point accuracy is $e p s=2.2204 e-16$, we probably have cumulative round-off errors since interface terms are much smaller than internal ones. The direct solver has a pivoting strategy that permits to obtain good quality solutions even on matrices having a poor condition number. But we are probably near the limits when using such a resolution strategy on large matrices issued from the proposed formulation.

When conducing the numerical tests our main aim was to validate the proposed alternative approach on few enough significative tests. On the scope of practical use, it is obvious that more intensive

\begin{tabular}{|l|r|r|r|r|r|r|r|}
\hline$h$ & $1 / 10$ & $1 / 20$ & $1 / 40$ & $1 / 70$ & $1 / 120$ & $1 / 220$ & $1 / 400$ \\
\hline \hline size & 253 & 784 & 2732 & 7791 & 21988 & 71961 & 234202 \\
\hline nnz & 3459 & 11218 & 40640 & 118241 & 338024 & 1115451 & 3647590 \\
\hline sparsity & $5.4 \%$ & $1.8 \%$ & $0.54 \%$ & $0.19 \%$ & $0.069 \%$ & $0.021 \%$ & $0.0066 \%$ \\
\hline
\end{tabular}

Table 2: Size, number of non zero entries, and sparsity rate as $h$ for the starfish domain 


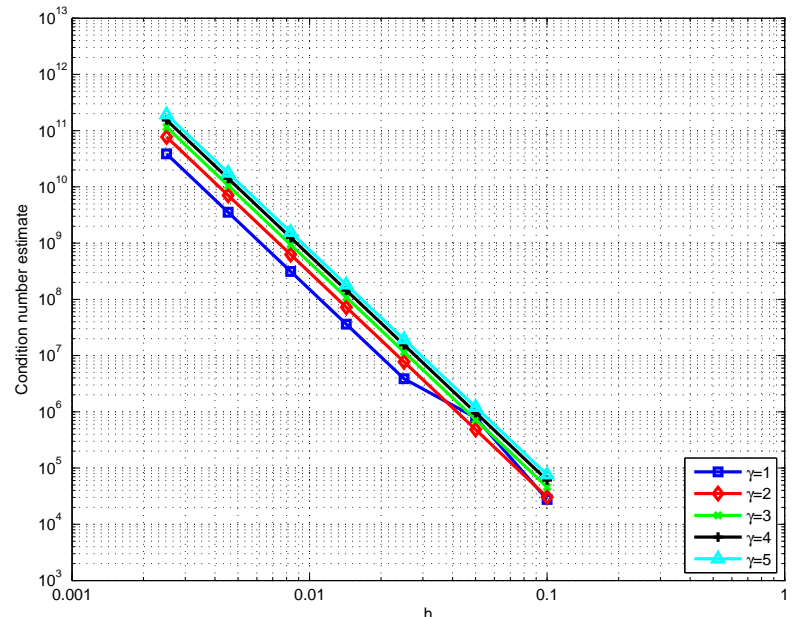

Figure 19: Condition number estimate for the matching square: $\gamma \in\{1,2,3,4,5\}$

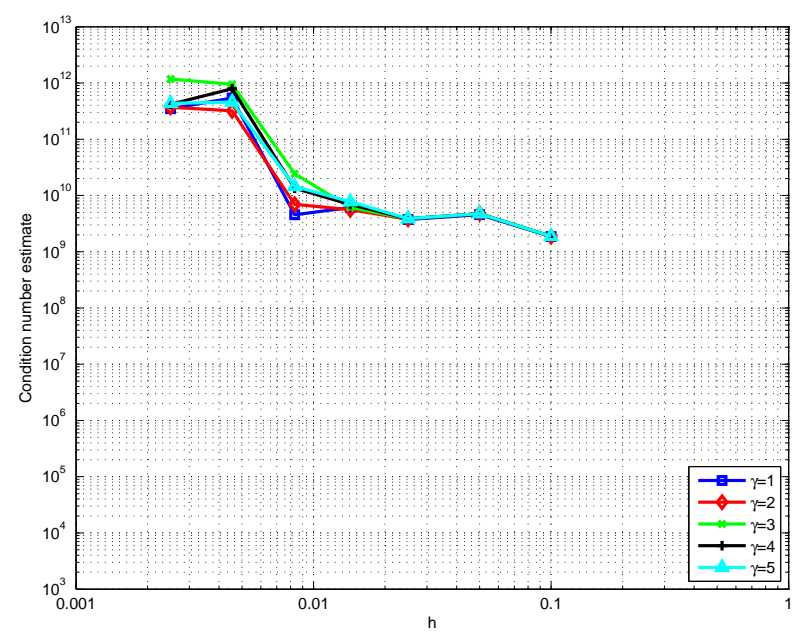

Figure 21: Condition number estimate for the starfish: $\gamma \in\{1,2,3,4,5\}$

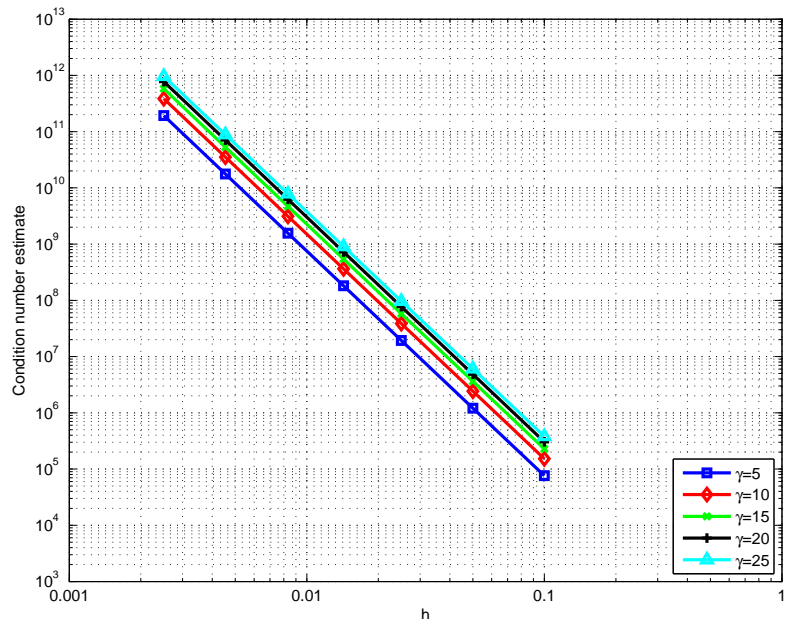

Figure 20: Condition number estimate for the matching square: $\gamma \in[5,25]$

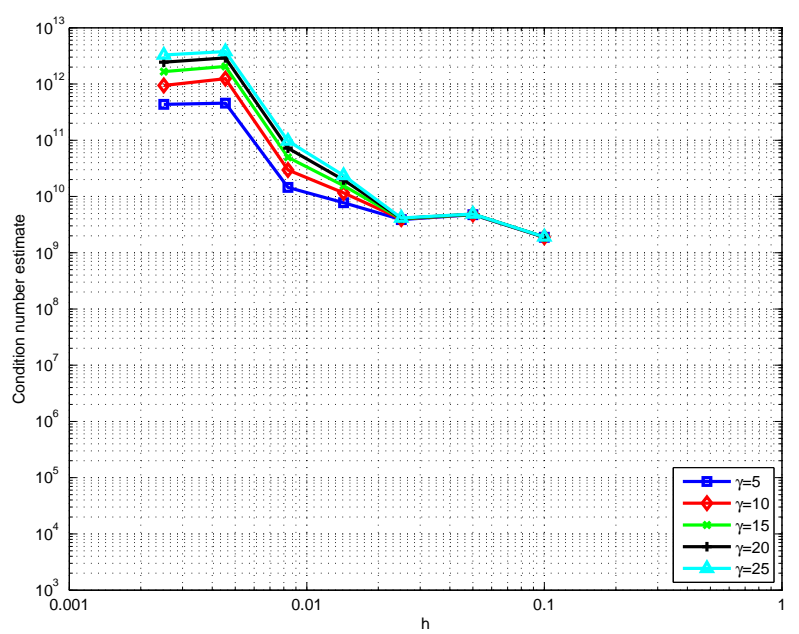

Figure 22: Condition number estimate for the starfish: $\gamma \in[5,25]$

experiments should be necessary in order to answer on questions relative to the choice of the parameter $\gamma$ and on the adequation between the intrinsic method and numerical solvers.

Conclusion We have presented a new method to solve a model boundary value problem with a structured mesh on a bounding box. This method is a variant of the usual Nitsche method with an interpretation in the continuous level, it is compatible with a level-set representation of the boundary but imposes restrictive conditions. The empirical results are by far better than the theoretical one. To obtain robustness and convergence, the procedure requires to adjust two 
parameters: a geometrical parameter $P_{c}$ and a Nitsche like coefficient $\gamma$. It seems that this coefficient can be chosen significantly smaller than Nitsche parameter.

\section{References}

[1] G. Allaire. Conception optimale de structures, volume 58 of Mathématiques $\& 3$ Applications (Berlin) [Mathematics \& Applications]. Springer-Verlag, Berlin, 2007. With the collaboration of Marc Schoenauer (INRIA) in the writing of Chapter 8.

[2] I. Babuška. Error-bounds for finite element method. Numer. Math., 16:322-333, 1970/1971.

[3] I. Babuška. The finite element method with Lagrangian multipliers. Numer. Math., 20:179$192,1972 / 73$.

[4] H. J. C. Barbosa and T. J. R. Hughes. Boundary Lagrange multipliers in finite element methods: error analysis in natural norms. Numer. Math., 62(1):1-15, 1992.

[5] J. W. Barrett and C. M. Elliott. Finite element approximation of the Dirichlet problem using the boundary penalty method. Numer. Math., 49(4):343-366, 1986.

[6] R. Becker, P. Hansbo, and R. Stenberg. A finite element method for domain decomposition with non-matching grids. M2AN Math. Model. Numer. Anal., 37(2):209-225, 2003.

[7] T. Belytschko, S. Xiao, and C. Parimi. Topology optimization with implicit functions and regularization. International Journal for Numerical Methods in Engineering, 57(8):1177-1196, 2003.

[8] J. Chessa and T. Belytschko. An enriched finite element method and level sets for axisymmetric two-phase flow with surface tension. Internat. J. Numer. Methods Engrg., 58(13):2041-2064, 2003.

[9] J. Chessa and T. Belytschko. An extended finite element method for two-phase fluids. Trans. ASME J. Appl. Mech., 70(1):10-17, 2003.

[10] J. Chessa, P. Smolinski, and T. Belytschko. The extended finite element method (XFEM) for solidification problems. Internat. J. Numer. Methods Engrg., 53(8):1959-1977, 2002.

[11] T. A. Davis. Algorithm 832: Umfpack v4.3-an unsymmetric-pattern multifrontal method. ACM Trans. Math. Softw., 30:196-199, June 2004.

[12] S. Dumont, O. Goubet, T. Ha-Duong, and P. Villon. Meshfree methods and boundary conditions. Internat. J. Numer. Methods Engrg., 67(7):989-1011, 2006.

[13] G. Dupire, J. Boufflet, M. Dambrine, and P. Villon. On the necessity of nitsche term. Applied Numerical Mathematics, In Press, Corrected Proof:-, 2010.

[14] R. Glowinski, T.-W. Pan, and J. Périaux. A fictitious domain method for Dirichlet problem and applications. Comput. Methods Appl. Mech. Engrg., 111(3-4):283-303, 1994.

[15] P. Hansbo. Nitsche's method for interface problems in computational mechanics. GAMM-Mitt., 28(2):183-206, 2005. 
[16] A. Henrot and M. Pierre. Variation et optimisation de formes, volume 48 of Mathématiques \& Applications (Berlin) [Mathematics 83 Applications]. Springer, Berlin, 2005. Une analyse géométrique. [A geometric analysis].

[17] P. Le Tallec and T. Sassi. Domain decomposition with nonmatching grids: augmented Lagrangian approach. Math. Comp., 64(212):1367-1396, 1995.

[18] J. A. Nitsche. Über ein Variationsprinzip zur Lösung von Dirichlet-problemen bei Verwendung von Teilräumen, die Keinen Randbedingungen unterworfen sind. Abh. Math. Sem. Univ. Hamburg, 36:9-15, 1971.

[19] A. Nouy, F. Schoefs, and N. Moës. X-sfem, a computational technique based on x-fem to deal with random shapes. European Journal of Computational Mechanics, 16(2):277-293, 2007.

[20] S. Osher and R. Fedkiw. Level set methods and dynamic implicit surfaces, volume 153 of Applied Mathematical Sciences. Springer-Verlag, New York, 2003.

[21] J. A. Sethian. Level set methods and fast marching methods, volume 3 of Cambridge Monographs on Applied and Computational Mathematics. Cambridge University Press, Cambridge, second edition, 1999. Evolving interfaces in computational geometry, fluid mechanics, computer vision, and materials science.

[22] R. Stenberg. On some techniques for approximating boundary conditions in the finite element method. J. Comput. Appl. Math., 63(1-3):139-148, 1995. International Symposium on Mathematical Modelling and Computational Methods Modelling 94 (Prague, 1994).

[23] R. Stenberg. Mortaring by a method of J. A. Nitsche. In Computational mechanics (Buenos Aires, 1998), pages CD-ROM file. Centro Internac. Métodos Numér. Ing., Barcelona, 1998.

[24] N. Sukumar, N. Moës, B. Moran, and T. Belytschko. Extended finite element method for threedimensional crack modeling. International Journal for Numerical Methods in Engineering, 48(11):1549-1570, 2000.

[25] M. E. Taylor. Pseudodifferential operators, volume 34 of Princeton Mathematical Series. Princeton University Press, Princeton, N.J., 1981.

[26] V. Thomée. Galerkin finite element methods for parabolic problems, volume 25 of Springer Series in Computational Mathematics. Springer-Verlag, Berlin, 1997. 